\title{
Radiation assurance for the space environment
}

\author{
Janet L. Barth, Senior Member, IEEE, Kenneth A. LaBel, Member, IEEE, Christian Poivey, Member, IEEE
}

\begin{abstract}
The space radiation environment can lead to extremely harsh operating conditions for spacecraft electronic systems. A hardness assurance methodology must be followed to assure that the space radiation environment does not compromise the functionality and performance of space-based systems during the mission lifetime. The methodology includes a definition of the radiation environment, assessment of the radiation sensitivity of parts, worst-case analysis of the impact of radiation effects, and part acceptance decisions which are likely to include mitigation measures.
\end{abstract}

\section{Introduction}

The Sun emits time-varying magnetic fields, plasmas, and energetic particles. This solar variability drives changes in the interplanetary environment which then interacts with the Earth's magnetic field and outermost atmosphere to produce changes in the nearEarth space environment. The space environment and its solar-induced changes interact with spacecraft and instrument components and can cause anomalies resulting in loss of data, degradation of capability, service outages, and, in extreme cases, the loss of spacecraft. The most effective time to prevent spacecraft anomalies is during the pre-launch phases when risk can be minimized through technology selection and system design. For most missions, some level of "residual risk" must be assumed due to cost constraints, increasing complexity of space systems, unknowns in the space environment, and/or unknowns in space environment effects mechanisms. Possible consequences of the residual risk on spacecraft health and safety and on degradation of service must be evaluated and mitigated by setting operational guidelines for spacecraft operators and working with operators to determine how to use the guidelines effectively.

The interaction of the space environment with spacecraft components induces a broad range of effects. The effects include degradation of materials, thermal changes, contamination, excitation, spacecraft glow, charging, communication and navigation errors and dropouts, radiation damage and radiation-induced background interference. This paper will focus on radiation effects.

J. Barth and K. LaBel are with NASA/Goddard Space Flight Center, Flight Data Systems and Radiation Effects Branch, Code 561.4, Greenbelt, MD 20771 USA

(email: Janet.L.Barth@nasa.gov)

C. Poivey is with SGT, Inc. at NASA/Goddard Space

Flight Center, Flight Data Systems and Radiation

Effects Branch, Code 561.4, Greenbelt, MD 20771 USA
Radiation accelerates the aging of the electronic parts and material and can lead to degradation of electrical performance; it can also create transient phenomena on parts. Such damage at the part level can induce damage or functional failure at electronic box, subsystem, and system levels. A rigorous methodology is needed to ensure that the radiation environment does not compromise the functionality and performance of the electronics during the system life. This methodology is called hardness assurance. It consists of those activities undertaken to ensure that the electronic piece parts placed in the space system perform to their design specifications after exposure to the space radiation environment. It deals with system requirements, environmental definitions, part selection, part testing, shielding, and radiation tolerant design. All these elements must play together in order to produce a system tolerant to the radiation environment within the level of risk that is acceptable for the mission.

\section{Hardness Assurance Overview}

Figure 1 gives an overview of the radiation hardness assurance process. A complete description is given by Poivey [1]. This process is iterative. It starts with toplevel estimations of the radiation environment and then the assessment of radiation levels is refined as needed to assure hardness. To validate the most sensitive parts, in-depth analysis of the measured part response to radiation testing, analysis of how the part is used in the electronic design, and allowances for operating conditions are required. The major activities in the hardness assurance program are:

Step I - Description of the mission radiation environment and definition of the radiation levels within the spacecraft

Step 2 - Assessment of the radiation sensitivity of the parts based on radiation databases and relevant radiation tests

Step 3 - Worst Case Analysis (WCA) of the impact of the radiation effect taking into account the system and circuit design

Based on the results from Steps $I$ and 2, the overall equipment and spacecraft worst-case performance over the mission length is estimated, taking into account radiation effects, aging and other causes of degradation. By combining the system application of each part and its radiation response, a radiation failure level can be determined.

\section{Step 4 - Part categorization}

The radiation level at which each part fails is compared to its mission radiation level or the radiation requirements and a decision is made concerning the 
hardness of all devices in the system. The part categorization is the key activity of a hardness assurance program.

During the lifetime of a mission's design and development, multiple variables change. These include:

- updates to parts list

- $\quad$ revised spacecraft layout

- revised mission requirements such as mission duration

- addition of new payloads, or

- the discovery of new radiation effects information.

Due to these and other factors, many of the steps in the approach may be revisited throughout the mission's radiation hardness assurance program.

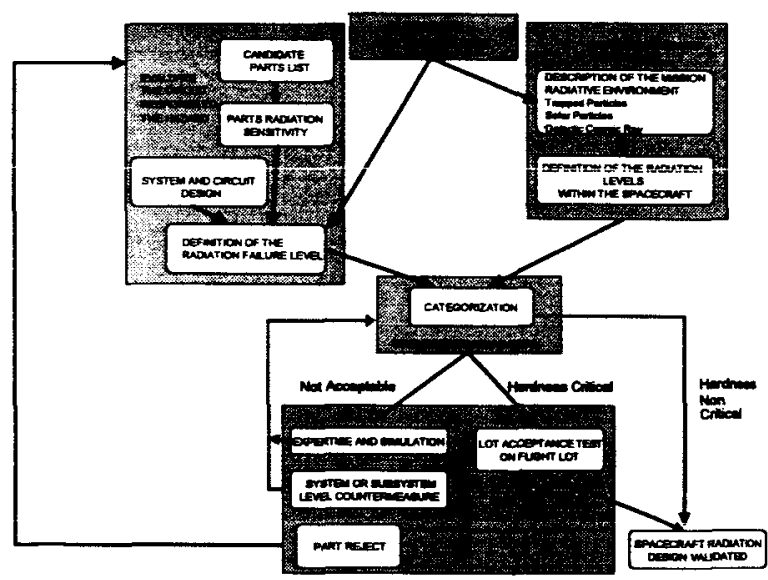

Figure 1: Overview of the radiation hardness assurance program

\section{Description of the Radiation Environment}

The characteristics of the radiation environment are highly dependent on the type of mission (date, duration and orbit) so the first step of hardness assurance is to understand the space radiation environment that is specific to the mission. The natural space radiation environment can be classified into two populations, 1) the transient particles which include protons and heavier ions of all of the elements of the periodic table, and 2) the trapped particles which include protons, electrons and heavier ions. The transient radiation consists of galactic cosmic ray (GCR) particles and particles from solar events (such as, coronal mass ejections and flares). The solar eruptions periodically produce energetic protons, alpha particles, heavy ions, and electrons. Table 1 lists the maximum energy of the radiation particles that are used for spacecraft assessments. Particles exist at higher energy levels
(TeVs), however not at flux levels that are a concern for spacecraft effects. The table shows that much of the environment is high-energy; therefore, shielding is not effective for many types of radiation effects. More complete descriptions of the radiation environment can be found in References 2, 3, and 4 .

Table 1: Maximum Energies of Particles Used in Assessments of Radiation Effects on Spacecraft

\begin{tabular}{|c|c|}
\hline Particle Type & Maximum Energy \\
\hline Trapped Electrons & 10 s of MeV \\
\hline $\begin{array}{c}\text { Trapped Protons \& Heavier } \\
\text { Ions }\end{array}$ & 100 s of MeV \\
\hline Solar Protons & $100 \mathrm{~s}$ of MeV \\
\hline Solar Heavy Ions & $\mathrm{GeV}$ \\
\hline Galactic Cosmic Rays & $\mathrm{GeV}$ \\
\hline
\end{tabular}

\section{A. Transient Populations}

Figure 2 is a plot of measurements of interplanetary abundances of the Carbon-NitrogenOxygen (CNO) group of ions as a function of time. The slowly varying low levels of particles are the GCR population. The effect of the 11-year solar cycle is evident with the peak GCR populations occurring near solar minimum. Superimposed on the GCR population are the unpredictable, sudden rises in the flux levels due to solar storms. Galactic and solar particles penetrate the Earth's magnetosphere and are particularly hazardous to satellites in polar, highly elliptical, and geostationary (GEO) orbits. Because the solar particle levels are extreme, there is increasing awareness of the need for a capability to forecast the occurrence of these events.

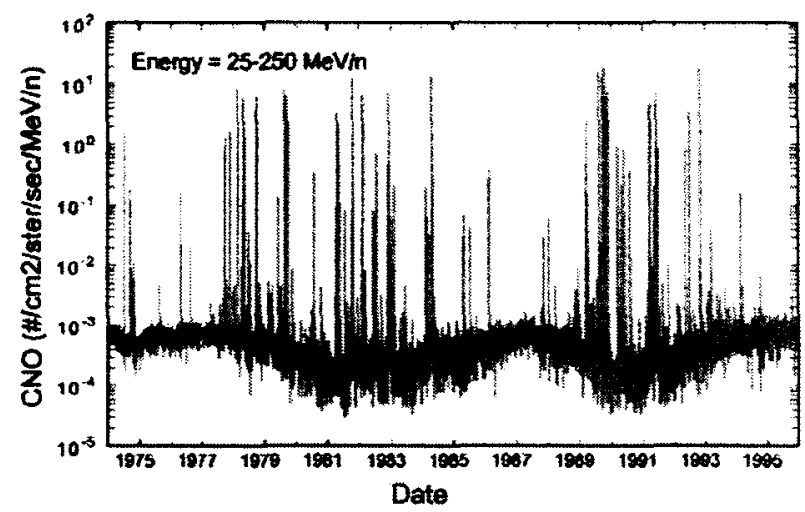

Figure 2: Measurement of interplanetary CNO ions from 1974 to 1996 - IMP-8 24-hour average mean exposure flux

\section{B. Trapped Populations}

Figure 3 is a drawing of the trapped proton and electron regions around the Earth known for their discoverer, James Van Allen. The E $>30 \mathrm{MeV}$ proton 
fluxes peak at approximately $2,500 \mathrm{~km}$ at the equator. The electrons are trapped in two regions, the inner and outer zones. The E $>2 \mathrm{MeV}$ electron fluxes peak at approximately 2,500 and $20,000 \mathrm{~km}$ at the equator. The particle levels and locations are highly dependent on particle energy, altitude, inclination, and the activity level of the Sun. The displacement of the Earth's magnetic field from the center and the tilt of the magnetic axis cause a dip in the magnetic field over the South Atlantic Ocean, resulting in a bulge in the underside of the inner belt. This region $(\sim 300$ to 1200 $\mathrm{km}$ ) is called the South Atlantic Anomaly (SAA). In spite of the SAA's reputation for plaguing spacecraft, the flux levels there are actually much lower than in the heart of the radiation belts.

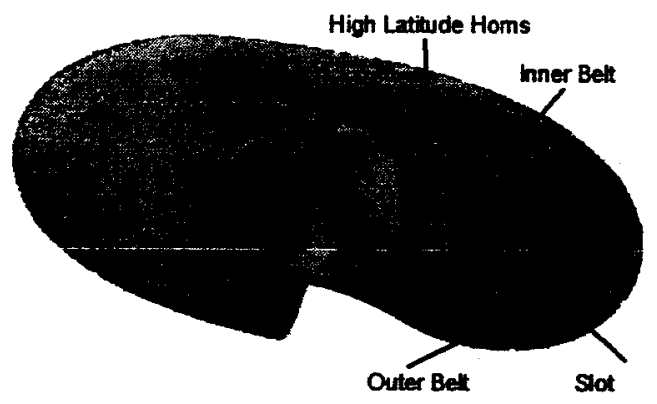

Figure 3: Drawing of the trapped particle populations

The trapped particle population is highly dynamic, especially in the slot region between the inner and outer zones and in the outer zone. Storms can induce sudden variations in particle levels that are several orders of magnitude higher than the average populations. Figure 4 is a plot of measurements of trapped electrons which show the extremely dynamic outer zone at $\mathrm{L}>$ 2.8 and the slot region $(2<\mathrm{L}<3)$ filling with storm electrons. Due to their complex distribution and dependence on long- and short-term solar variability, the trapped particle populations are difficult to model and forecast.

To avoid exposure to the trapped radiation environment, missions operate in low earth orbits (below the belts) or in geostationary (outside of the highly energetic, intense trapped proton and electron regions). To increase observation capabilities, there is growing interest in flying in middle earth orbit (MEO) regions. Due to the lack of experience in MEO, little information is available on the accuracy of radiation environment models in MEO.

\section{Models of the Space Radiation Environment}

Standard models of the trapped and transient radiation environments are used to estimate levels for missions. Poivey [1] provides the most recent review of available radiation environment models.

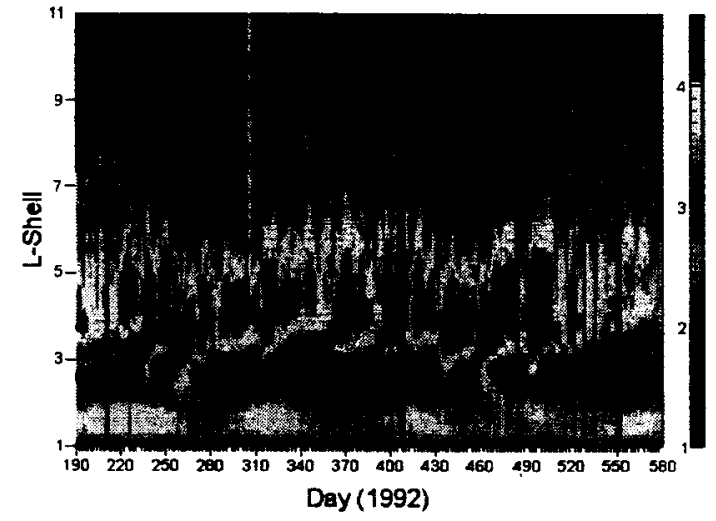

Figure 4: Measurement of trapped electrons which show the extremely dynamic outer zone at $L>2.8$ and the slot region $(2<\mathrm{L}<3)$ filling with storm electrons $\mathrm{E}>0.4 \mathrm{MeV}-$ SAMPEX/PIADC

\section{Step 1 - Definition of Radiation Environments}

The hardness assurance process begins with definitions of the radiation environment that can be used for estimates of radiation induced damage levels and interference rates. The definitions need to be specific to the part location. Interactions of the radiation environment cause a wide range of radiation effects. Those that are important to consider for instrument and spacecraft design fall roughly into four categories: degradation from total ionizing dose (TID), degradation from displacement damage, and single event effects (SEEs). Radiation environment definitions must be specific to the models that simulate the radiation effects and, in most cases, each effect requires different environment parameters. The required model inputs will be reviewed below.

\section{A. Total Ionizing Dose}

Total ionizing dose (TID) is cumulative long-term damage caused by solar and trapped protons and trapped electrons. In microelectronics, TID causes threshold shifts, leakage current, and timing skews. The effect first appears as parametric degradation of the device and can ultimately result in functional failure. When a manufacturer advertises a part as "rad-hard", he is almost always referring to its TID characteristics. Rad-hard does not usually imply that the part is hard to non-ionizing dose or SEEs.

It is possible to reduce TID with shielding material that absorbs most electrons and lower energy protons. As shielding is increased, shielding effectiveness decreases because of the difficulty in slowing down the higher energy protons. TID levels are calculated from the solar and trapped particles incident on the spacecraft and are given as a function of dose versus aluminum 
shield thickness for a simple geometry. The geometry model generally used is the solid sphere, which gives an upper bound for the dose inside an actual spacecraft. This value is used as a top-level requirement. Figure 5 plots the total ionizing dose for several spacecraft orbits. As parts are characterized for their radiation hardness, it often becomes necessary to refine the total dose requirement. This will be discussed in more detail in Section VII.

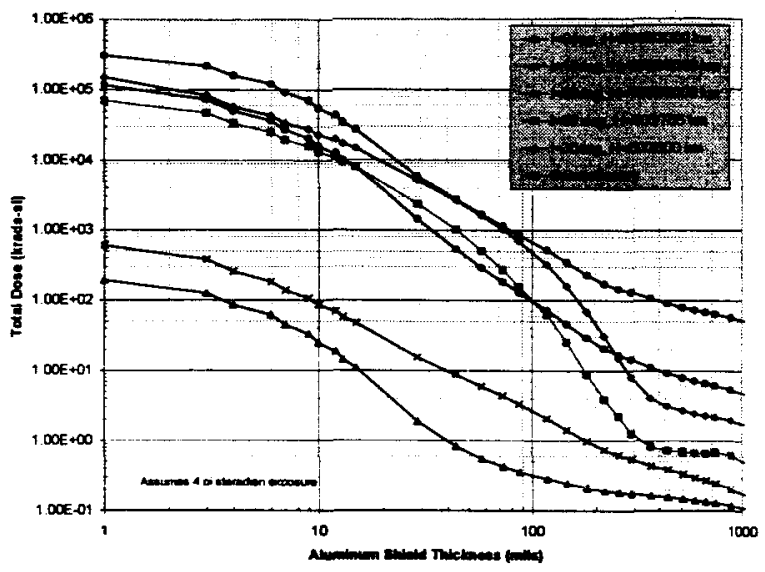

Figure 5: Total ionizing dose for near-earth orbiting spacecraft for 1 year in orbit

\section{B. Displacement Damage Dose}

Degradation also occurs when high-energy particles displace atoms from the material lattice structure. Displacement damage dose (DDD) is also called bulk damage or degradation via non-ionizing energy loss (NIEL) in materials. It accumulates as materials are exposed to solar and trapped protons and trapped electrons. Secondary neutrons that are produced in shielding materials or the atmosphere or neutrons from external sources can also cause displacement damage. The particles produce defects that result in charge transfer degradation. It affects the performance of optocouplers (often a component in power devices), solar cells, CCDs, and linear bipolar devices. Shielding has limited effectiveness against the damage. Coverglasses over solar cells reduce damage by absorbing the low energy particles, but they cannot absorb the high-energy protons. Shielding is not usually effective for optoelectronic components because the high-energy protons penetrate most realistic spacecraft electronic enclosures. CCDs are heavily shielded, however, secondary particle production can become problematic. Displacement damage effects are calculated from total particle fluence levels as shown for a Low Earth Orbit (LEO) in Figure 6. The example in Figure 6 also shows that adding shielding has little effect on the high-energy trapped protons.

\section{Single Event Effects}

Single event effects are due to ionization when a single charged particle passes through a sensitive junction of an electronic device. They are caused by galactic cosmic rays and solar heavy ions, but for some devices, trapped and solar protons can induce SEEs. SEEs can be induced by direct ionization by protons, but in most instances, proton induced effects are the result of secondary particles that are produced when the proton collides with a nucleus of the material in the device. Some SEEs are non-destructive, as in the case of single event upsets (SEUs), single event transients (SETs), multiple bit errors (MBEs), single event hard errors (SHEs), single event transients (SETs), etc. SEEs can also be destructive as in the case of single event latchups (SELs), single event gate ruptures (SEGRs), and single event burnouts (SEBs).

The cosmic ray and solar heavy ion single event effects are evaluated using the linear energy transfer (LET) metric. LET is a measure of the energy deposited along the path of the particle. Figure 7 shows LET values at 1 AU (applicáblé to geostatióary orbits). Rather than LET, the proton energy spectra are more appropriate for the evaluation of proton-induced events (see Figure 6). The spectra should be given for a range of operating conditions, i.e., peak fluxes, average fluxes, and sustained exposures (during solar events and passes through the SAA).

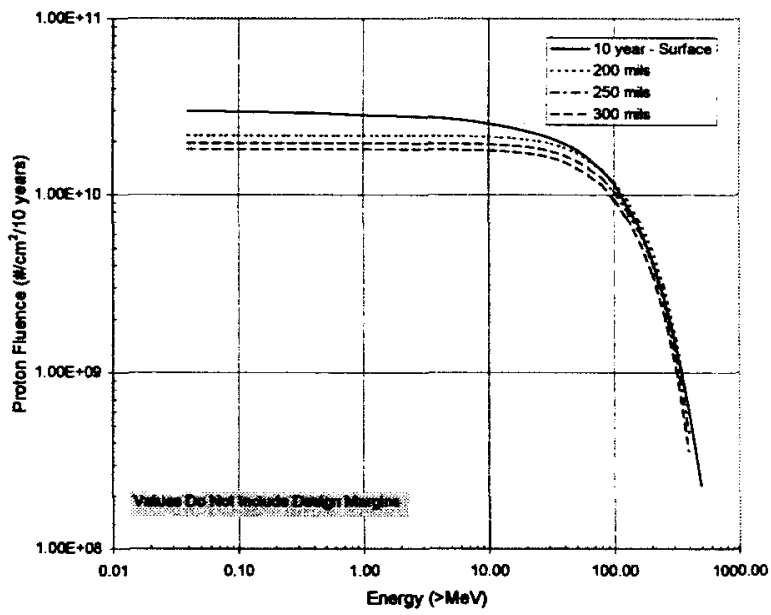

Figure 6: Surface incident and shielded trapped protons responsible for displacement damage. Fluences are orbitintegrated, trapped protons for a $29 \mathrm{deg}, 590 \mathrm{~km}$ circular orbit for a 10-year mission. 


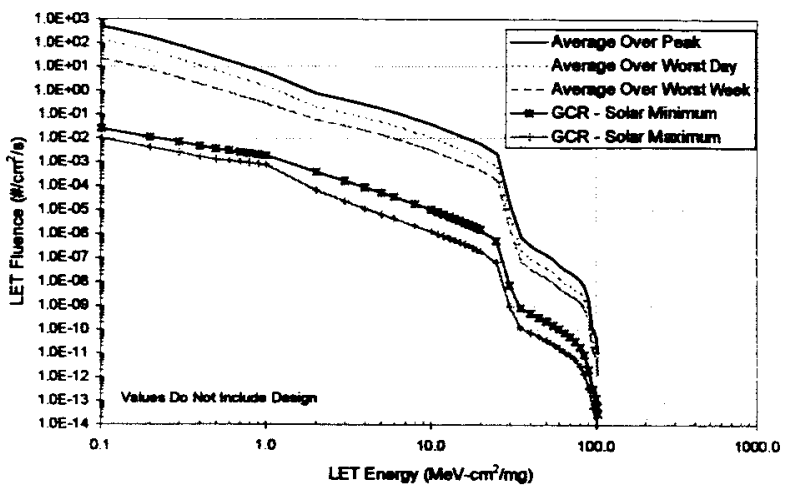

Figure 7: Integral LET spectra are shown for interplanetary galactic cosmic ray and solar ions at $1 \mathrm{AU}$, hydrogen through uranium. The fluences are shielded by 100 mils of aluminum.

\section{Step 2 - Assessment of the Radiation Sensitivity of Parts}

Knowledge of the radiation sensitivity of all parts to be used on the instruments and spacecraft is essential to the success of the overall hardness assurance program. Radiation testing is expensive and should be avoided when possible, therefore, maximum use of part characterizations performed on other programs is desirable. A careful literature search and analysis can circumvent unnecessary tests.

If there are no available radiation data or if the test data are not sufficient, radiation test data must be obtained. The objectives of radiation testing are threefold:

- Research: Understand the mechanisms of interaction of the radiation with electronic materials and how these effects relate to device failure.

- Characterization: Characterize the response of specific device types and technologies for use in part selection for specific system application.

- Hardness Assurance: Determine the acceptability of production lots.

In order to obtain the database needed for these three objectives, the space radiation environment must be simulated in the laboratory. Although attempts are often made to duplicate the space environment to the greatest extent possible by irradiating with the same particle type, energies, and fluxes encountered in space, more often the dominant effect of the radiation is simulated with a convenient radiation source to reduce the cost and technical problems.

One important "rule of thumb" is to "Test as you fly" which means simulating the operating conditions of the devices. The failure mechanisms of many microelectronic devices exposed to radiation are a strong function of the operating bias, operating mode (standby or active), and temperature. Devices are usually characterized under a variety of test conditions in order to find the worst case operating conditions.

The temperature of many space electronics systems is controlled to be within a range of $0-80{ }^{\circ} \mathrm{C}$. Failure levels within this range usually do not vary significantly from room temperature, where most radiation testing is performed. There are some space applications, however, where temperature extremes are encountered, such as cryogenic electronics for certain detectors and high temperatures for some space power systems. In these cases, the failure levels can be significantly different from those measured at room temperature, and the testing must be performed at the appropriate temperature.

\section{A. Use of Existing Radiation Data}

There are many sources of radiation data on semiconductor devices and microcircuits. Many agencies offer radiation effects databases on the web:

- NASA-GSFC radiation effects data base (hup://radhome.gsfc.nàsa.gōvitoup.himi)

- NASA-JPL radiation effects data base (http://radnet.jpl.nasa.gov)

- DTRA ERRIC radiation effects database (http://erric.dasiac.com/)

- ESA radiation effects database (https://escies.org/)

The IEEE NSREC data-workshop proceedings, IEEE Transactions on Nuclear Science, and RADECS Proceedings are also useful sources of information. Some manufacturers make available the radiation data about their products.

The existence of radiation data on a device does not necessarily indicate the device's acceptability for a mission. Most of the available data are out of date, not well documented (in terms of bias conditions, radiation source characteristics or measurement techniques), or are peculiar to nuclear weapons rather than the space environment. For many commercial parts, the design is changed, the feature size is shrunk, and the process is improved in a continual effort to improve performance, yield and reliability. Many of these changes affect the radiation response.

General guidelines for acceptability of archive data are:

- The tests have been performed with the approved US or European test procedures (see Ref. 1). For linear devices, if the part has been tested to TID at high dose rate, retesting is recommended.

- A sufficient number of parts have been tested.

- The tested part has the same technology as the part that will be used for flight. 
- For TID data, if the lot date code is different, testing is recommended, but may be waived if sufficient process information is gathered. Acceptable conditions for a testing waiver are similar Lot (or date code) with known process changes, or devices for which the die topology and substrate characteristics are known to be the same as for an older lot of devices.

- The electrical parameters and performances important for the application have been tested.

- The bias conditions during testing are worse or equivalent to the application.

The two last steps are perhaps the most difficult. A good example is evaluating test data for SET in linear circuits. In this case, an understanding of what the sensitive SET characteristics (amplitude and duration) are in a design's application is required.

\section{B. Testing to Obtain Radiation Data}

Frequently, it is necessary to perform device testing. The previous section showed the difficulty in using existing test data. Even when test data are applicable, the testing may not have covered all of the possible radiation effects to which the device may be susceptible or may not cover worst-case operating conditions.

\section{Total Ionizing Dose Testing}

Total ionizing dose testing is performed by exposing a device to an ionizing radiation environment and by measuring its electrical performance for a variety of operating conditions. There are two approaches that can be used to characterize the response: step stress and influx testing. Step-stress testing is performed by first characterizing the electrical performances of the device, exposing it to a fixed dose of ionizing radiation, and then measuring again the electrical parameters to determine their change. To determine the device response versus total dose, the test is performed with different samples of the same type at a number of accumulated dose levels. In-flux testing is performed by continually measuring the device response as it is being irradiated. The step stress approach is usually more convenient and much more widely used.

Many linear bipolar circuits exhibit enhanced low dose rate sensitivity (ELDRS) where the damage is greater when parts are exposed to lower dose rates in space than the higher exposure rates in ground tests [5, $6,7,8]$. For those circuits, standard test procedures, such as, the MIL-STD1019.5 and the ESA/SCC 22900, may be non-conservative, because the accelerated aging test does not work [9]. This is why bipolar technology is specifically excluded from the TM1019.5 accelerated aging test. Several accelerated tests for bipolar microcircuits have been suggested. Generally, they propose high dose rate irradiation tests at elevated temperature $[10,11,12]$, but to date no successful standard test procedure has been found to bound the part response of all types of bipolar linear circuits.

\section{Displacement Damage Testing}

Displacement damage testing for the space environment is performed using the step stress approach. Although a significant amount of transient annealing can occur immediately after a short pulse of radiation, this effect is not a factor for space particle flux levels. The permanent damage is stable at room temperature, hence no significant annealing occurs between irradiation steps. It has also been shown that bias has little effect on the permanent displacement damage. This allows irradiation to be performed passively [13]. Therefore displacement damage testing consists of simply characterizing the electrical performance of the part, exposing to an irradiation source, without bias, to a fixed particle fluence and characterizing it after irradiation to determine the parameter degradation. However, for photonics devices, the degradation may be application dependant. For example, Reed showed this application dependence for optocouplers in [14]. For these devices active measurements, that match the application, are recommended.

The radiation source used is generally a monoenergetic proton beam, and the part is irradiated to a fluence greater than the mission displacement damage dose or equivalent fluence established with the NIEL. It is very important to choose adequately the test energy. For low quantities of shielding, a low energy (i.e., 10 $\mathrm{MeV}$ ) is adequate because it represents best the environment. For higher shielding, a higher energy is needed (i.e., $60 \mathrm{MeV}$ ), because most of the damage results from protons higher than $10 \mathrm{MeV}$ [13]. Reed recommends testing optocouplers at multiple energies because of the inconsistency between experimental determination of damage factors and theoretical calculations with NIEL [15].

Protons are also heavily ionizing with a larger fraction of the energy loss going into ionization, therefore the effects will include both displacement and ionizing dose damages. The test total deposited dose needs to be calculated and the results compared to a Co-60 TID test to sort out failure mechanisms.

For solar cells the radiation source is generally a mono-energetic electron ( $1 \mathrm{MeV}$ is a standard value) or proton ( $10 \mathrm{MeV}$ is a standard value) beam, and the part is irradiated to a fluence greater than the mission equivalent fluence established with the NIEL or with the damage equivalent models. 


\section{Single Event Effects Testing}

Single Event Effects testing is performed using the in-flux test method. The microcircuit is electrically exercised by a tester, and the errors are counted during irradiation. High-energy galactic cosmic rays and solar event heavy ions are simulated with low energy ions available in particle accelerators. The index of quality used is the amount of energy lost per unit length of track, the linear energy transfer (LET). As the SEE sensitive regions of many microcircuits are relatively thin (several $\mu \mathrm{m}$ ), ground testing is conducted using ions with lower energies than GCR or solar event heavy ions, but with similar LET. The energy range at the SEE facilities commonly used is of the order of several $\mathrm{MeV} / \mathrm{u}$ ( $\mathrm{u}$ is the atomic mass unit) and the penetration range of ions is about from $30 \mu \mathrm{m}$ for the heaviest particles to $100 \mu \mathrm{m}$ for the lightest particles. Shortcomings of SEE ground testing have been discussed in several papers $[16,17,18,19]$. Generally the LET concept gives a conservative estimate of part SEE sensitivity. As the range of ions available at ground level is low, these tests are performed under vacuum for low energy beams and the device package in front of the die is removed. At each value of LET the bit error rate is measured by counting a statistically significant number of errors. The SEE cross section in $\mathrm{cm}^{2}$ (or $\mathrm{cm}^{2} / \mathrm{bit}$ ) is the ratio of the measured number of errors to the ion fluence in particle $/ \mathrm{cm}^{2}$. Figure 8 shows an example of an SEU cross-section obtained from a heavy ion test.

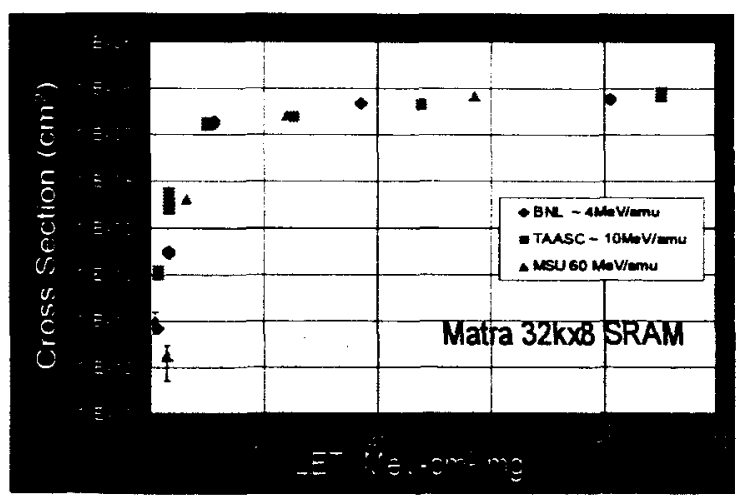

Figure 8: SEU cross-section obtained from a heavy ion test. The low LET threshold ( $<5 \mathrm{MeV}-\mathrm{cm}^{2} / \mathrm{mg}$ ) suggests that the device may be susceptible to proton induced SEUs.

The energy range of protons in space is directly available on synchrotron accelerators at ground level. The testing is similar to the heavy ion test, but for protons it is the SEE cross section versus proton energy curve that is measured. The criterion for proton testing is based on heavy ion threshold LET for the type of event that is studied. For silicon parts, the proton cross section is negligible when threshold LET as measured using heavy ion is larger than $15 \mathrm{MeVcm}^{2} / \mathrm{mg}$; no proton testing is required in this case.

Because proton induced SEEs involve spallation products created after a nuclear reaction between proton and the device's atoms, it is possible to estimate the proton sensitivity based on heavy ion data. Specific models $[20,21,22,23,24,25]$ exist which allow an estimation of the proton induced SEE cross section curves based on heavy ion data. This approach can be used to get a first idea of the device sensitivity, but if this device is critical for the application, a proton testing characterization is recommended.

Test standards have been developed in the US (JEDEC Test standard 57 or US ASTM F1192-90) and in Europe (ESA/SCC25100). The JEDEC test standard 57 is only valid for heavy ions $(\mathrm{Z}>2)$. The ESA/SCC 25100 is applicable for both heavy ions and protons testing.

Each type of SEE requires special testing techniques to obtain valid test data. These techniques have necessarily evolved with technology advancements, such as, large commercial memories and microprocessors. With the advancements, difficulties are encountered in assessing the effects due to device complexities. Also, more exotic single event effects are observed in advanced technologies, such as, single event transients that are dependent on bias conditions and "microlatches" exhibited by very small changes in current in circuits. These issues are reviewed in Ref. 1 and Ref. 26 in more detail.

\section{Step 3 - Perform Worst Case Analysis (WCA) of the Impact of the Radiation Effect}

The radiation levels for each sensitive part are defined by the methods described in the previous section. For a given part type, the radiation level is defined as the worst-case radiation level. Considering the individual part radiation sensitivities, the radiation environment, and the system/subsystem design, an analysis of the system/subsystem response is performed. The design radiation analysis is part of the design Worst Case Analysis (WCA) that combines the effects of radiation, temperature and parts aging. Circuit WCA is needed on each engineering subsystem and science instrument in order to demonstrate that the design will work in its environment under the most stressful operating conditions (data rates, voltages, switching transients,...). The design engineer usually performs the circuit WCA.

As an example for TID, Figure 9 shows the degradation of the offset voltage $V_{i o}$ of an operational amplifier PM155 versus total ionizing dose. The data have been collected on 8 parts from the same lot and 
the average has been calculated. If we consider the specification limit of $2 \mathrm{mV}$, the failure level is about 40 krad. If we consider an acceptable limit for a given design of $4 \mathrm{mV}$ (this means that the acceptable $V_{\text {io }}$ for this design will be higher taking into account the temperature variation and the part aging), $\mathrm{Rf}$ will be about $75 \mathrm{krad}$ for this particular application.

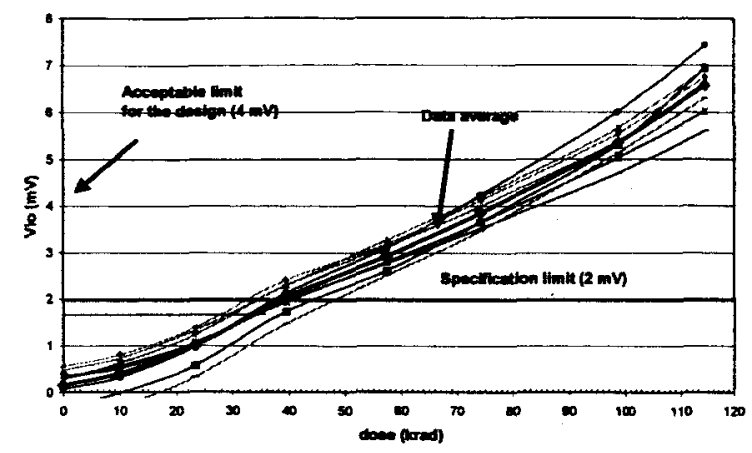

Figure 9: Degradation of the offset voltage of a PM155 operational amplifier versus total dose

The approach for displacement damage analysis is similar to TID. The test data and the design WCA allow the definition of the failure level $R f$. Then, the failure level is compared to the DDD or the mission equivalent fluence to define the Radiation Design Margin. Generally, the design margins requirements for DDD and TID are the same. Devices sensitive to Displacement Damage are also sensitive to TID. For proton dominated space environments, DDD proton testing can deliver sufficient TID to estimate both the DDD and TID induced degradation. Otherwise, the effects of TID and DDD need to be combined for the design WCA. Reed proposes such an approach to estimate the CTR degradation for a specific mission DDD and TID [27].

SEE requirements depend on the functions the devices perform. Many SEEs are different for different device types. For example, memories will exhibit different conditions than linear devices. In addition, SEEs may present functional impacts by propagating through the design and impacting other areas. These two conditions make each single event problem different in terms of failure mode and effect. SEE analysis is most effectively supported by viewing a design or system from the perspective of the function(s) performed.

For SEE analysis, the SEE data are compared first to the radiation environment and then a Single Event Effect Criticality Analysis (SEECA) is performed [28]. Functions may then be categorized into "criticality classes" or categories of differing severity of SEE occurrence. Many times, most or all of the functions performed by a design or system are considered critical to a mission. The operational impact of SEEs in critical functions may be unacceptable. For these designs, usually either no single event effects, or a very small probability of SEE occurrences, are permitted. When considering a subsystem, some components may not be SEE-critical, while data storage memories may tolerate SEEs if utilizing error correction schemes. Both of these functions are located in the Data System.

In general, one might consider three criticality groups for Single Event Upset: error-functional, errorvulnerable, and error-critical. Functions in the errorfunctional groups may be unaffected by SEUs, whether this immunity is due to an implemented error-correction scheme or redundancy, and a large probability of SEU at the device level may be acceptable. Functions in the error-vulnerable group might be those for which the risk of a low probability is acceptable. Functions in the error-critical group are functions where SEU is unacceptable.

\section{VII.Step 4 - Part categorization}

The Radiation Design Margin (RDM) is used to select the acceptance category in which each part falls. RDM is defined as the ratio of the part failure level to the part radiation environment. When the part hardness greatly exceeds the system requirements, the part is not hardness critical and can be used in the application without any further action.

For each radiation sensitive part, the Radiation Design Margin (RDM) is defined and then each part could be classified as Hardness non critical, Hardness critical or not acceptable. For the parts belonging to the two last categories, risk reduction actions are taken.

The US Space Working Group (SPWG) has developed two formalisms for categorization: the Design Margin Breakpoint (DMBP) and the Part Categorization Criterion (PCC) [29]. The DMBP is a qualitative approach recommended for systems with moderate requirements according to the guideline document for ionizing dose and neutron hardness assurance MIL-HDBK 814. The application of the DMBP method for categorization is shown in Table 2.

Table 2: Application of the DMBP method for part categorization [29]

\begin{tabular}{|c|c|}
\hline RDM & Categorization \\
\hline$<1-2$ & Unacceptable \\
\hline $1-2<$ RDM $<10-100$ & Hardness Critical \\
\hline RDM $>10-100$ & Hardness non critical \\
\hline
\end{tabular}

When the part hardness is lower than the radiation level $(R D M<1)$, the part cannot be used as is and risk reduction tasks must be performed. Risk reduction tasks can include: 
- Investigation: A more accurate estimation of the radiation level (e.g. using a 3 D MonteCarlo code to calculate the total dose level received by the part) may allow a reduction of the radiation requirement. On the other hand, a complementary radiation test closer to the application conditions may also increase the radiation failure level of the part.

- System or equipment level countermeasures: Countermeasures can be implemented to either increase the acceptable sensitivity level of the part or reduce the radiation environment: additional shielding at component level (spot shielding) or at box level (additional thickness of box cover), switching of redundant component or function, error correction system, specific memory organization, latchup protection circuitry, etc.

- Part replacement: The part is replaced by another part having a higher radiation tolerance.

When the part hardness is not significantly higher than the system requirements, the part is considered radiation hardness critical. In order to minimize the risk associated with the spread of device hardness between manufacturing lots (and also within the same manufacturing lot), flight lot parts can be tested during procurement.

In cases where parts cannot meet the top-level TID requirement and a "harder" part cannot be substituted, it is beneficial to employ more accurate methods of determining the dose exposure to qualify the parts. One such method is solid angle sectoring/3-dimensional ray tracing. An accurate computer model of the electronics box and/or spacecraft is produced and average path lengths through sectors are calculated. With that information, total doses can be obtained for any location. Changing the model and recalculating the total dose can accurately evaluate the value of dose mitigation measures. Doses obtained by sectoring methods must be verified for $5-10 \%$ of the sensitive locations with full Monte Carlo simulations of particle trajectories through the structure for many histories. Often more rigorous shielding analysis will provide the required RDM to qualify the part. If this is not the case, spot shielding can be used or the box with the sensitive part can be moved to a more protected position on the spacecraft provided the analysis has been done early in the program.

More rigorous shielding analysis is often useful for bounding the DDD problems, however, the practical limitations of mass and volume make it difficult to add adequate shielding to absorb high-energy protons. For sensitive, mission critical optical components, it is critical to begin ground radiation testing and subsequent investigations and system level countermeasures as early as possible in the program.

Risk reduction for SEEs is more complex. If a part replacement cannot be found (as is often the case), system level countermeasures are required. Destructive SEE conditions (Single Event Latch-up, Single Event Gate Rupture, Single Event Burnout) may or may not be recoverable depending on the individual device's response. Hardening from the system level is difficult at best, and in most cases not particularly effective. Generally, parts with a non-negligible destructive SEE rate should not be used. On a case-by-case basis use of these parts with adequate circumvention methods could be authorized.

For non-destructive SEEs, mitigation is more effective. Once the acceptable event rates are defined, they are compared to the device event rates. Generally a Radiation Design Margin of at least 2 is required. If a part is found to be unacceptable, the alternatives are to redesign the system (to increase the acceptable error rate) or substitute a harder part. Another alternative could also be the reallocation of the acceptable error rates. Mitigation requires 1) careful device level analysis which will determine the effect of the SEU on device performance, 2) analysis of circuit operation and performance to determine how the SEU sensitive device is being used, and 3) system level analysis to assess error propagation.

Examples of SEE mitigation methods are presented in [30].

\section{Management Of Hardness Assurance}

There are many ways of managing the process of hardness assurance described in the previous chapters. The options are a centralized radiation effects management or a devolved management, in which subsystem engineers are responsible for all environmental constraints. The latter option is generally used for space systems. Requirements are reflected through a Radiation Environment specification and a Radiation Hardness specification, which define the external environment, the techniques to be used and the radiation design margin required. At NASA-GSFC a lead radiation engineer is assigned to each space flight project. The RHA engineering process [26] is viewed in a manner similar to that used by a mechanical or a thermal engineer who is assigned for the life of a project. With a single point of contact for all project radiation issues (environment, device selection, testing) each program has a radiation effects expert responsible for ensuring performance in the radiation environment. By participating early in flight programs, the radiation effects engineer may contribute to cost reduction strategies. 


\section{Conclusions}

The two main activities of a radiation hardness assurance program are the definition of the radiation environment at the part level and the definition of the part failure level. As more and more TID sensitive parts are used, a top-level requirement is usually not sufficient except for programs with low TID requirements. An accurate spacecraft modeling and a 3D sectoring/ray trace or Monte Carlo radiation analysis allows a significant reduction of the TID requirement. Another advantage of having a spacecraft model is the ability to analyze the effectiveness of mitigation techniques, e.g., moving boxes to locations that offer more protection or adding spot shielding to parts.

The radiation characterization is the first step of the definition of the part failure level. Then, the part radiation sensitivity is compared to the part uses in the different applications and the impact at the circuit level, box level, subsystem level, and system level. Design mitigation techniques allow the use of radiation sensitive parts. All these activities affect the spacecraft and electronic box layout, the system design, and cven system operations. The radiation hardness assurance process is no longer confined to the part level. Radiation hardness has to be taken into account at all the stages of the system development. Taking into account the hardness assurance in the early phases of a program development will allow reducing significantly the costs of hardness assurance.

\section{References}

[1] C. Poivey, "Radiation Hardness Assurance for Space Systems," Notes from the 2002 IEEE Nuclear and Space Radiation Effects Short Course, Phoenix, AZ.

[2] J. L. Barth, "Modeling Space Radiation Environments," Notes from the 1997 IEEE Nuclear and Space Radiation Effects Short Course, Snowmass, CO.

[3] C. Dyer, "Space Radiation Environment Dosimetry," Notes from the 1998 IEEE Nuclear and Space Radiation Effects Short Course, Newport Beach, CA.

[4] J. Mazur, Notes from the 2002 IEEE Nuclear and Space Radiation Effects Short Course, Phoenix, AZ.

[5] J. Beaucour, T. Carriere, A. Gach, D. Laxague, and P. Poirot, "Total Dose Effects on Negative Voltage Regulator," IEEE Trans. Nuc. Sci, vol. 41, n6, pp. 2420-2426, Dec. 1994.

[6] S. Mc Clure, R. L. Pease, W. Will, and G. Perry, "Dependence of Total Dose Response of Bipolar Linear Microcircuits on Applied Dose Rate," IEEE Trans. Nuc. Sci, vol. 41, n6, pp. 2544-2549, Dec. 1994.

[7] A. H. Johnston, G. M. Swift, and B. G. Rax, "Total Dose Effects in Conventional Bipolar Transistors and Linear Integrated Circuits," IEEE Trans. Nuc. Sci., vol. 41, ${ }^{\circ}$ 6, pp. 2427-2436, Dec. 1994.

[8] A. H. Johnston, B. G. Rax, and C. I. Lee, "Enhanced Damage in Linear Bipolar Integrated Circuits at Low
Dose Rates," IEEE Trans. Nuc. Sci., vol. 42, nº 6 , pp 1650-1659, Dec. 1995.

[9] R. L. Pease, "Total Dose Issues for Microelectronics in Space Systems," IEEE Trans. Nucl. Sci., vol. $43, \mathrm{n}^{\circ} 2$, pp. 442-452, Apr. 1996.

[10] S.C. Witczak, R. D. Schrimpf, D. M. Fleetwood, K. F. Galloway, R. C. Lacoe, D. C. Mayer, J. M. Puhl, R. L. Pease, and J. Suehle, "Hardness Assurance Testing of Bipolar Junction Transistors at Elevated Irradiation Temperatures," IEEE Trans. Nuc. Sci., vol. 44, nº, pp. 1989-2000, Dec. 1997.

[11] R. L. Pease, M. Gehlhausen, "Elevated Temperature Irradiation of Bipolar Linear Microcircuits," IEEE Trans. Nucl. Sci., vol. S43, nº, p. 3161-3166, Dec. 1996.

[12] L. Bonora, J. P. David, "An attempt to define conservative conditions for total dose evaluation of bipolar ICs," IEEE Trans. Nucl. Sci., vol. 44, n6, pp. 1974-1980, Dec. 1997.

[13] C. Marshall, and P. Marshall, "Proton Effects and Test Issues for Satellite Designers, part B: Displacement Effect," 1999 IEEE NSREC short course, Norfolk, Jul. 1999.

[14] R. A. Reed, P. W. Marshall, A. H. Johnston, J. L. Barth, C. J. Marshall, K. A. LaBcl, M. D'O'dine, H. S. Kim, and M. A. Carts, "Emerging Optocoupler Issues with Energetic Particle-Induced Transients and Permanent Radiation Degradation," IEEE Trans. Nuc. Sci., vol. 45, n6, pp. 2833-2841, Dec. 1998.

[15] R. A. Reed, "Guideline for Ground Radiation Testing and Using Optocouplers in the Space Radiation Environment," NASA-GSFC report, Mar. 2002.

[16] E. G. Stassinopoulos, G. J. Brucker, "Shortcomings in Ground Testing, Environment simulations, and Performance Predictions for Space Applications," RADECS 1991 Proceedings, pp. 3-16, 1992.

[17] R. Koga, "Single Event Effect Ground Test Issues," IEEE Trans. Nucl. Sci., vol. 43, n², pp. 661-670, Apr. 1996.

[18] S. Duzellier, R. Ecoffet, "Recent Trends in Single Event Effect Ground Testing," IEEE Trans. Nucl. Sci., vol. 43, n'2, pp. 671-677, Apr. 1996.

[19] C. Poivey, J. L. Barth, R. A. Reed. K. A. LaBel, E. Stassinopoulos, and M. Xapsos, "Implications of Advanced Microelectronics Technologies for Heavy Ion Single Event Effect (SEE) Testing," RADECS 2001 proceedings, Grenoble, France.

[20] J. G. Rollins, "Estimation of Proton Upset Rate from Heavy Ion Test Data," IEEE Trans. Nucl. Sci., vol. 37, n6, pp. 1961-1965, Dec. 1990.

[21] E.L. Petersen, "The Relationship of Proton and Heavy Ion Upset Thresholds," IEEE Trans. Nucl. Sci., vol. 39, n6, pp. 1600-1604, Dec. 1992.

[22] B. Doucin, T. Carriere, C. Poivey, P. Garnier, J. Beaucour, "Model of Single Event Upsets Induced by Space Protons in Electronic Devices," RADECS 1995 proceedings, pp. 402-408, 1996.

[23] P. Calvel, C. Barillot, P. Lamothe, R. Ecoffet, S. Duzellier, and D. Falguere, "An Empirical Model for Predicting Proton Induced Upset," IEEE Trans. Nucl. Sci., vol. 43, n6, pp. 2827-2832, Dec. 1996. 
[24] V.V. Miroshkin, M. G. Tverskoy, "A Simple Approach to SEU Cross Section Evaluation," IEEE Trans. Nucl. Sci.,vol. 45, n6, pp. 2884-2890, Dec. 1998.

[25] J. Barak, "Empirical Modeling of Proton Induced SEE Rates," IEEE Trans. Nucl. Sci., vol. 47, n³, pp. 545550, Jun. 2000.

[26] K. A. LaBel, A. H. Johnston, J. L. Barth, R. A. Reed, and C. E. Barnes, "Emerging Radiation Hardness Assurance (RHA) issues: A NASA Approach for Space Flight Programs," IEEE Trans. Nucl. Sci., vol. 45, n6, pp. 2727-2736, Dec. 1998.

[27] R. A. Reed, C. Poivey, P. W. Marshall, K. A. LaBel, C. J. Marshall, S. Kniffin, J. L. Barth, and C. Seidleck, "Assessing the Impact of the Space Radiation Environment on Parametric Degradation and SingleEvent Transients in Optocouplers," IEEE Trans. Nuc. Sci., vol. 48, n6, pp. 2202-2209, Dec. 2001.

[28] K. LaBel, "SEECA Single Event Effect Criticality Analysis, SEU propagation analysis: system level effects," http://radhome.gsfc.nasa.gov/radhome/papers/seecai.htm 1996.

[29] R. L. Pease, and D. R. Alexander, "Hardness Assurance for Space Systems Microelectronics," Rad. Phys. Chem., vol. 43, $\mathrm{n}^{\circ} 1 / 2$, pp. 191-204, 1994.

[30] K. LaBel, and M. Gates, "Single-Event-Effect Mitigation From a System Perspective," IEEE Trans. Nucl. Sci., vol. 43, n², pp. 654-660, Apr. 1996. 